\title{
DIFFERENCE EQUATIONS FOR HYPERGEOMETRIC POLYNOMIALS FROM THE ASKEY SCHEME. SOME RESULTANTS. DISCRIMINANTS. *
}

\author{
INNA NIKOLOVA ${ }^{\dagger}$
}

\begin{abstract}
It is proven that every sequence from the Askey scheme of hypergeomeric polynomials satisfies differential or difference equations of first order of the form $T p_{n}(x)=A_{n}(x) p_{n-1}(x)-$ $B_{n}(x) p_{n}(x)$, where $T$ is a linear degree reducing operator, which leeds to the fact that these polynomial sets satisfy relation of the form $p_{n}^{\prime}(x)=A_{n}(x) p_{n-1}(x)-B_{n}(x) p_{n}(x)$.
\end{abstract}

1. Introduction. It is shown that the set of polynomials from the Askey scheme of hypergeometric polynomials satisfy a differential or a difference equation of first order, which is generally nonhomogenious. Y. Chen and M. Ismail show in [2], that any sequence of polynomials, orthogonal w. r. t. a continuous measure of the type $w(x)=e^{-v(x)}$, where $v(x)$ is twice continuously differentiable and convex on a closed interval satisfy first order differential equation. Earlier versions of equation of the same form are in [1]. From the Askey scheme the result in [2] applies for Hermite polynomials, more generaly it applies in other cases not included in this scheme. In [6] it is shown that the convexity condition can be replaced with the condition that $v^{\prime}(x)$ is positive on the open interval of integration. So the results can be applied for the classical Laguerre and Jacobi polynomials. In this paper we consider all the cases from the Askey scheme of the hypergeometric polynomials in which the above results do not apply and prove that they satisfy a difference equation of the form:

$$
T p_{n}(\lambda(x))=A_{n}(x) p_{n-1}(\lambda(x))-B_{n}(x) p_{n}(\lambda(x))
$$

where $T$ is linear degree reducing operator and $A_{n}(x)$ and $B_{n}(x)$ are rational functions of $x$. The above result leads to the equation of the form:

$$
p_{n}^{\prime}(\lambda(x))=A_{n}(x) p_{n-1}(\lambda(x))-B_{n}(x) p_{n}(\lambda(x)),
$$

which is useful for the determination of the discriminants of the corresponding polynomial sets, since these polynomial sets satisfy three term recurrence relation and the theorem proven in [1] applies. However the practical usage of this theorem is very difficult.One can sertainly compute the discriminants of the above polynomial set till $n=4$.

The discriminants of the classical orthogonal polynomials of Jacobi, Hermite and Laguerre were computed by Stieltjes and Hilbert [10]. Closed forms of the discriminants of the rest of the polynomial sets in Askey scheme are not known. Similarly Ismail [5] showed that the computational procedure used for the classical orthogonal polynomials can be applied for any set of polynomials,generated by

$$
p_{n}(x)\left(a_{n} x+b_{n}\right) p_{n-1}(x)-c_{n} p_{n-2}(x),
$$

which satisfies a relation of the form:

$$
p_{n}^{\prime}(x)=A_{n}(x) p_{n-1}(x)-B_{n}(x) p_{n}(x) .
$$

\footnotetext{
* Received December 20, 2002; accepted for publication November 26, 2003.

$\dagger$ Institute of Computer Communication systems, Bulgarian Academy of Science, Akad. G. Bonchev street bl 2, Sofia 1113, Bulgaria (inna@agatha.iac.bg). I would like to say thank you to Dr. M. E. H. Ismail, whose idea was [8]. Without working on it this paper would have been impossible.
} 
in terms of the functions $A_{n}(x)$ and the coefficients of the three term recurrence relation. In this paper we prove that the discriminant of a sequence of polynomials generated by

$$
p_{n}(\lambda(x))=\left(a_{n} \lambda(x)+b_{n}\right) p_{n-1}(\lambda(x))-c_{n} p_{n-2}(\lambda(x))
$$

where

$$
\lambda(x)=x(x+\gamma+\delta+1)
$$

can be explicitly obtained in terms of the recurrence coefficients and the functions $A_{n}(x)$ in (2). Therefore equations of the forms (4) and (2) are of some interest. We would like to emphasize to the fact that the results in [2] and [6] are not applicable to the polynomial sets form the Askey scheme except for the classical polynomials. Here we show that using the difference equations one can compute some resultants between the polynomial itself and the polynomials w.r.t.x obtained by applying the operator $T$ to the polynomial of the variable $\lambda(x)$.

\section{Difference equations.}

THEOREM 2.1. Let $p_{n}(\lambda(x))$ is a sequence of polynomials from the Askey scheme, where $\lambda(x)=x(x+\gamma+\delta+1)$ or $\lambda(x)=x$, then

$$
T\left(p_{n}(\lambda(x))\right)=A_{n}(x) p_{n-1}(\lambda(x))-B_{n}(x) p_{n}(\lambda(x))
$$

and

$$
T\left(p_{n}(\lambda(x))\right)=-C_{n}(x) p_{n}(\lambda(x))+D_{n}(x) p_{n+1}(\lambda(x))
$$

Proof. The fact that the classical orthogonal polynomials of Hermite, Laguerre and Jacobi satisfy differential equation of first order is well known [10]. These facts can be obtained also from [2] and [6]. The cases for Meixner Crawtchouck, Meixner and Hahn polynomials are considered in earlier paper by Ismail, Simeonov and me cite[Is:Ni:Si] .

- Difference equation for dual Hahn polynomials. By the definition given in [9]:

$$
R_{n}(\lambda(x))=\sum_{k=0}^{n} \frac{(-n)_{k}(-x)_{k}(x+\gamma+\delta+1)_{k}}{k !(\gamma+1)_{k}(-N)_{k}}
$$

Consider the following operator

$$
\Delta R_{n}(\lambda(x))=\frac{R_{n}(\lambda(x+1))-R_{n}(\lambda(x))}{\lambda(x+1)-\lambda(x)} .
$$

Since

$$
\begin{aligned}
& (-x-1)_{k}(x+\gamma+\delta+2)_{k}-(-x)_{k}(x+\gamma+\delta+1)_{k} \\
& =-k(2 x+\gamma+\delta+2)(-x)_{k-1}(x+\gamma+\delta+2)_{k-1},
\end{aligned}
$$


it follows that

$$
\begin{aligned}
\Delta R_{n}(\lambda(x))= & -\frac{1}{(x+\gamma+\delta+1)} \sum_{k=0}^{n-1} \frac{(-n)_{k+1}(-x)_{k}(x+\gamma+\delta+1)_{k+1}}{k !(\gamma+1)_{k+1}(-N)_{k+1}} \\
= & -\frac{1}{(x+\gamma+\delta+1)} \\
& \times \sum_{k=0}^{n-1} \frac{(-n)_{k+1}(-x)_{k}(x+\gamma+\delta+1)_{k+1}[(-x+k)-(-N+k)]}{k !(\gamma+1)_{k+1}(-N)_{k+1}(-x+N)} \\
= & \frac{1}{(x+\gamma+\delta+1)(x-N)} \sum_{k=0}^{n-1} \frac{(-n)_{k+1}(-x)_{k+1}(x+\gamma+\delta+1)_{k+1}}{k !(\gamma+1)_{k+1}(-N)_{k+1}}- \\
& -\frac{1}{(x+\gamma+\delta+1)(x-N)} \\
& \times \sum_{k=0}^{n-1} \frac{(-n)_{k+1}(-x)_{k}(x+\gamma+\delta+1)_{k+1}(-x+k-\gamma-k-1)}{k !(\gamma+1)_{k+1}(-N)_{k}(-x-\gamma-1)}
\end{aligned}
$$

Let us denote by

$$
\zeta_{n, k}=\frac{(-n)_{k}(-x)_{k}(x+\gamma+\delta+1)_{k}}{k !(\gamma+1)_{k}(-N)_{k}} .
$$

Then

$$
\begin{aligned}
& \Delta R_{n}(\lambda(x)) \\
& =\frac{1}{(x+\gamma+\delta+1)(x-N)} \sum_{k=0}^{n} k \zeta_{n, k} \\
& +\frac{1}{(x+\gamma+\delta+1)(x-N)(x+\gamma+1)} \sum_{k=0}^{n-1} \frac{(-n)_{k+1}(-x)_{k+1}(x+\gamma+\delta+1)_{k+1}}{k !(\gamma+1)_{k+1}(-N)_{k}} \\
& -\frac{1}{(x+\gamma+\delta+1)(x-N)(x+\gamma+1)} \sum_{k=0}^{n} \frac{(-n)_{k+1}(-x)_{k}(x+\gamma+\delta+1)_{k+1}}{k !(\gamma+1)_{k}(-N)_{k}} \\
& =\frac{1}{(x+\gamma+\delta+1)(x-N)(x+\gamma+1)} \sum_{k=0}^{n} \zeta_{n, k} k(x+\gamma+1) \\
& +\frac{1}{(x+\gamma+\delta+1)(x-N)(x+\gamma+1)} \sum_{k=0}^{n} \zeta_{n, k} k(-N-1+k) \\
& -\frac{1}{(x+\gamma+\delta+1)(x-N)(x+\gamma+1)} \sum_{k=0}^{n} \zeta_{n, k}(-n+k)(x+\gamma+\delta+k+1) \\
& =\frac{1}{(x+\gamma+\delta+1)(x-N)(x+\gamma+1)} \sum_{k=0}^{n} \zeta_{n, k} G(k, x, n, \alpha, \beta, \gamma, \delta) \text {, }
\end{aligned}
$$

where

$$
\begin{aligned}
G(k, x, n, \gamma, \delta) & =k(x+\gamma+1)+k(-N-1+k)+(n-k)(x+\gamma+\delta+k+1) \\
& =n(x+\gamma+\delta+1)-k(N+1-n+\delta)
\end{aligned}
$$


Using

$$
(-n)_{k} k=n(-n)_{k}-n(-n+1)_{k}
$$

we see that

$$
\begin{aligned}
\Delta R_{n}(\lambda(x))= & \frac{n(N+1+\delta-n)}{(x+\gamma+\delta+1)(x-N)(x+\gamma+1)} R_{n-1}(\lambda(x)) \\
& +\frac{n(x+\gamma-N+n)}{(x+\gamma+\delta+1)(x-N)(x+\gamma+1)} R_{n}(\lambda(x)) .
\end{aligned}
$$

REMARK. Similar equation holds for the operator $\frac{\nabla R_{n}(x)}{\nabla \lambda(x)}=\frac{R_{n}(\lambda(x))-R_{n}(\lambda((x-1))}{\lambda(x)-\lambda(x-1)}$.

- Difference equation for Racah polynomials. By definition the Racah polynomials are given by[9]:

$$
R_{n}(\lambda(x))=\sum_{k=0}^{n} \frac{(-n)_{k}(\alpha+\beta+n+1)_{k}(-x)_{k}(x+\gamma+\delta+1)_{k}}{k !(\alpha+1)_{k}(\beta+\delta+1)_{k}(\gamma+1)_{k}}
$$

Consider the operator (9) and using (10) we obtain that

$$
\begin{aligned}
& \Delta R_{n}(\lambda(x)) \\
= & -\frac{1}{(x+\gamma+\delta+1)} \\
& \times \sum_{k=0}^{n-1} \frac{(-n)_{k+1}(n+\alpha+\beta+1)_{k+1}(-x)_{k}(x+\gamma+\delta+1)_{k+1}(-x+k-\beta-\delta-1-k)}{k !(\alpha+1)_{k+1}(\beta+\delta+1)_{k+1}(\gamma+1)_{k+1}(-x-\beta-\delta-1)} \\
= & \frac{1}{(x+\gamma+\delta+1)(x+\beta+\delta+1)} \\
& \times \sum_{k=0}^{n-1} \frac{(-n)_{k+1}(n+\alpha+\beta+1)_{k+1}(-x)_{k+1}(x+\gamma+\delta+1)_{k+1}}{k !(\alpha+1)_{k+1}(\gamma+1)_{k+1}(\beta+\delta+1)_{k+1}} \\
& -\frac{1}{(x+\gamma+\delta+1)(x+\beta+\delta+1)} \\
& \times \sum_{k=0}^{n-1} \frac{(-n)_{k+1}(n+\alpha+\beta+1)_{k+1}(-x)_{k}(x+\gamma+\delta+1)_{k+1}(-x+k-\gamma-k-1)}{k !(\alpha+1)_{k+1}(\beta+\delta+1)_{k}(\gamma+1)_{k+1}(-x-\gamma-1)}
\end{aligned}
$$

Let us use the following notation:

$$
\zeta_{n, k}=\frac{(-n)_{k}(n+\alpha+\beta+1)_{k}(-x)_{k}(x+\gamma+\delta+1)_{k}}{k !(\alpha+1)_{k}(\beta+\delta+1)_{k}(\gamma+1)_{k}} .
$$


DIFFERENCE EQUATIONS FOR HYPERGEOMETRIC POLYNOMIALS...

Then

$$
\begin{aligned}
& \Delta R_{n}(\lambda(x)) \\
&= \frac{1}{(x+\gamma+\delta+1)(x+\beta+\delta+1)} \sum_{k=0}^{n} \zeta_{n, k} k \\
& \times \sum_{k=0}^{n-1} \frac{1}{(x+\gamma+\delta+1)(x+\beta+\delta+1)(x+\gamma+1)} \\
&-\frac{1}{(x+\gamma+\delta+1)(x+\beta+\delta+1)(x+\gamma+1)} \\
& \times \sum_{k=0}^{n-1} \frac{(-n)_{k+1}(n+\alpha+\beta+1)_{k+1}(-x)_{k}(x+\gamma+\delta+1)_{k+1}(-x+k-\alpha-1-k)}{k !(\alpha+1)_{k+1}(\beta+\delta+1)_{k}(\gamma+1)_{k}(-x-\alpha-1)} \\
&= \frac{1}{(x+\gamma+\delta+1)(x+\beta+\delta+1)} \sum_{k=0}^{n} \zeta_{n, k} k \\
&+ \frac{1}{(x+\gamma+\delta+1)(x+\beta+\delta+1)(x+\gamma+1)} \sum_{k=0}^{n} \zeta_{n, k}(\beta+\delta+k) k \\
&+\frac{1}{(x+\gamma+\delta+1)(x+\beta+\delta+1)(x+\gamma+1)(\alpha+x+1)} \\
& \times \sum_{k=0}^{n-1} \frac{(-n)_{k+1}(n+\alpha+\beta+1)_{k+1}(-x)_{k+1}(x+\gamma+\delta+1)_{k+1}}{k !(\alpha+1)_{k+1}(\beta+\delta+1)_{k}(\gamma+1)_{k}} \\
& \times \frac{1}{(x+\gamma+\delta+1)(x+\beta+\delta+1)(x+\gamma+1)(\alpha+x+1)} \\
& \sum_{k=0}^{n} \frac{(-n)_{k+1}(n+\alpha+\beta+1)_{k+1}(-x)_{k}(x+\gamma+\delta+1)_{k+1}}{k !(\alpha+1)_{k}(\beta+\delta+1)_{k}(\gamma+1)_{k}}
\end{aligned}
$$

Under the notation

$$
f(x)=\frac{1}{(x+\gamma+\delta+1)(x+\beta+\delta+1)(x+\gamma+1)(\alpha+x+1)},
$$

it follows that:

$$
\frac{\Delta R_{n}(\lambda(x))}{\Delta \lambda(x)}=f(x) \sum_{k=0}^{n} \zeta_{n, k} G(k, x, n, \alpha, \beta, \gamma, \delta)
$$

where

$$
\begin{aligned}
G(k, x, n, \alpha, \beta, \gamma, \delta)= & (x+\gamma+1)(\alpha+x+1) k \\
+ & (\alpha+x+1)(\beta+\delta+k) k+k(\beta+\delta+k)(\gamma+k) \\
- & (-n+k)(n+\alpha+\beta+1+k)(x+\gamma+\delta+k+1) .
\end{aligned}
$$

After some computational work we compute that:

$$
G(k, x, n, \alpha, \beta, \gamma, \delta)=-k^{2}+k[x(x+\gamma+\delta+1)+M]+N(x) .
$$


where we used the notation:

$$
\begin{gathered}
M=\alpha \beta-\beta \delta+\gamma \delta+n(n+\alpha+\beta+1) \\
N(x)=n(n+\alpha+\beta+1)(x+\gamma+\delta+1) .
\end{gathered}
$$

Let us denote by

$$
\zeta_{n-1, k}^{*}=\frac{(-n+1)_{k}(\alpha+\beta+n+1)_{k}(-x)_{k}(x+\gamma+\delta+1)_{k}}{k !(\alpha+1)_{k}(\beta+\delta+1)_{k}(\gamma+1)_{k}} .
$$

Using again (11)we see that

$$
\begin{aligned}
\Delta R_{n}(\lambda(x))= & -f(x) \sum_{k=0}^{n} \zeta_{n, k} k^{2}+n f(x)[x(x+\gamma+\delta+1)+M] R_{n}(\lambda(x)) \\
& -n f(x)[x(x+\gamma+\delta+1)+M] \sum_{k=0}^{n} \zeta_{n-1, k}^{*}+f(x) N(x) R_{n}(\lambda(x)) .
\end{aligned}
$$

But

$$
(-n)_{k} k^{2}=n^{2}(-n)_{k}-n^{2}(-n+1)_{k}-n(-n+1)_{k} k
$$

and

$$
-\sum_{k=0}^{n} \zeta_{n, k} k^{2}=-n^{2} R_{n}\left(\lambda(x)+n^{2} \sum_{k=0}^{n} \zeta_{n-1, k}^{*}+n \sum_{k=0}^{n-1} k \zeta_{n-1, k}^{*}\right.
$$

Substituting (14) into (13) we get

$$
\begin{aligned}
\Delta R_{n}(\lambda(x))= & n f(x)(n+\alpha+\beta+1)(x+\gamma+\delta+1) R_{n} \\
+ & n f(x)[x(x+\gamma+\delta+1)+\alpha \beta-\beta \delta+\gamma \delta+n(\alpha+\beta+n)] R_{n} \\
& -n f(x)[x(x+\gamma+\delta+1)+\alpha \beta-\beta \delta+\gamma \delta+n(\alpha+\beta+n)] \sum_{k=0}^{n} \zeta_{n-1, k}^{*} \\
& +n f(x) \sum_{k=0}^{n-1} \zeta_{n-1, k}^{*} k .
\end{aligned}
$$

Let us denote by

$$
\begin{aligned}
F(x)= & {[x(x+\gamma+\delta+1)+\alpha \beta-\beta \delta+\gamma \delta+n(\alpha+\beta+n)] \sum_{k=0}^{n} \zeta_{n-1, k}^{*} } \\
& -\sum_{k=0}^{n-1} \zeta_{n-1, k}^{*} k
\end{aligned}
$$

Claim that there are coefficients $C_{n}$ and $D_{n}$, not depending on $x$, such that $F(x)$ can be expressed as

$$
F(x)=C_{n} R_{n-1}(\lambda(x))+D_{n} R_{n}(\lambda(x))
$$


DIFFERENCE EQUATIONS FOR HYPERGEOMETRIC POLYNOMIALS...

This statement can be proven easily. Indeed:

$$
\begin{gathered}
x(x+\gamma+\delta+1)(-x)_{k}(x+\gamma+\delta+1)_{k} \\
=-(-x)_{k+1}(x+\gamma+\delta+1)_{k+1}+k(\gamma+\delta+1)(-x)_{k}(x+\gamma+\delta+1)_{k}+k^{2}(-x)_{k}(x+\gamma+\delta+1)_{k}
\end{gathered}
$$

The substituting of (18) into (16) implies that

$$
\begin{aligned}
& F(x) \\
& =-\sum_{k=0}^{n-1} \frac{(-n+1)_{k}(\alpha+\beta+n+1)_{k}(-x)_{k+1}(x+\gamma+\delta+1)_{k+1}}{k !(\alpha+1)_{k}(\beta+\delta+1)_{k}(\gamma+1)_{k}} \\
& +\sum_{k=0}^{n-1} \frac{(-n+1)_{k}(\alpha+\beta+n+1)_{k}(-x)_{k}(x+\gamma+\delta+1)_{k} k(\gamma+\delta+1)}{k !(\alpha+1)_{k}(\beta+\delta+1)_{k}(\gamma+1)_{k}} \\
& +\sum_{k=0}^{n-1} \frac{(-n+1)_{k}(\alpha+\beta+n+1)_{k}(-x)_{k}(x+\gamma+\delta+1)_{k} k^{2}}{k !(\alpha+1)_{k}(\beta+\delta+1)_{k}(\gamma+1)_{k}} \\
& +[\alpha \beta-\beta \delta+\gamma \delta+n(\alpha+\beta+n)] \\
& \times \sum_{k=0}^{n-1} \frac{(-n+1)_{k}(\alpha+\beta+n+1)_{k}(-x)_{k}(x+\gamma+\delta+1)_{k}}{k !(\alpha+1)_{k}(\beta+\delta+1)_{k}(\gamma+1)_{k}} \\
& -\sum_{k=0}^{n-1} \frac{(-n+1)_{k}(\alpha+\beta+n+1)_{k}(-x)_{k}(x+\gamma+\delta+1)_{k} k}{k !(\alpha+1)_{k}(\beta+\delta+1)_{k}(\gamma+1)_{k}} \\
& =-\sum_{k=0}^{n} \frac{(-n+1)_{k-1}(\alpha+\beta+n+1)_{k-1}(-x)_{k}}{k !(\alpha+1)_{k}(\beta+\delta+1)_{k}(\gamma+1)_{k}} \\
& \times(x+\gamma+\delta+1)_{k} k(\alpha+k)(\beta+\delta+k)(\gamma+k) \\
& +\sum_{k=0}^{n} \frac{(-n+1)_{k-1}(-n+k)(\alpha+\beta+n+1)_{k-1}(\alpha+\beta+n+k)}{k !(\alpha+1)_{k}(\beta+\delta+1)_{k}(\gamma+1)_{k}} \\
& \times(-x)_{k}(x+\gamma+\delta+1)_{k} k(\gamma+\delta+1) \\
& +\sum_{k=0}^{n} \frac{(-n+1)_{k-1}(-n+k)(\alpha+\beta+n+1)_{k-1}(\alpha+\beta+n+k)(-x)_{k}(x+\gamma+\delta+1)_{k} k^{2}}{k !(\alpha+1)_{k}(\beta+\delta+1)_{k}(\gamma+1)_{k}} \\
& +[\alpha \beta-\beta \delta+\gamma \delta+n(\alpha+\beta+n)] \\
& \times \sum_{k=0}^{n-1} \frac{(-n+1)_{k-1}(-n+k)(\alpha+\beta+n+1)_{k-1}(\alpha+\beta+n+k)(-x)_{k}(x+\gamma+\delta+1)_{k}}{k !(\alpha+1)_{k}(\beta+\delta+1)_{k}(\gamma+1)_{k}} \\
& -\sum_{k=0}^{n-1} \frac{(-n+1)_{k-1}(-n+k)(\alpha+\beta+n+1)_{k-1}(\alpha+\beta+n+k)(-x)_{k}(x+\gamma+\delta+1)_{k} k}{k !(\alpha+1)_{k}(\beta+\delta+1)_{k}(\gamma+1)_{k}} .
\end{aligned}
$$

Finally we see that

$$
F(x)=\sum_{k=0}^{n} \kappa_{n, k} P(k)
$$

where

$$
\kappa_{n, k}=\frac{(-n+1)_{k-1}(\alpha+\beta+n+1)_{k-1}(-x)_{k}(x+\gamma+\delta+1)_{k}}{k !(\alpha+1)_{k}(\beta+\delta+1)_{k}(\gamma+1)_{k}}
$$


and

$$
\begin{aligned}
P(k)= & -k(\alpha+k)(\beta+\delta+k)(\gamma+k)-(n-k)(\alpha+\beta+n+k) k(\gamma+\delta) \\
& -(n-k)(\alpha+\beta+n+k) k^{2} \\
& -(n-k)(\alpha+\beta+n+k)[\alpha \beta-\beta \delta+\gamma \delta+n(\alpha+\beta+n)]
\end{aligned}
$$

or

$$
\begin{aligned}
P(k)= & k[\beta(\alpha-\delta)(\alpha+\beta-\gamma)+n(\alpha+\beta+n)(\alpha+\beta-\gamma-\delta)] \\
& -n(\alpha+\beta+n)[\alpha \beta-\beta \delta+\gamma \delta+n(\alpha+\beta+n)]
\end{aligned}
$$

So we see that:

$$
\begin{aligned}
F(x)= & {\left[-n^{2}(\alpha+\beta+n)^{2}-n(\alpha+\beta+n)(\alpha \beta-\beta \delta+\gamma \delta)\right] \sum_{k=0}^{n} \kappa_{n, k} } \\
& -[n(\alpha+\beta+n)(\gamma+\delta-\alpha-\beta)+\beta(\alpha-\delta)(\gamma-\alpha-\beta)] \sum_{k=0}^{n} \kappa_{n, k} k
\end{aligned}
$$

In order (17) to be true there must be coefficients $C_{n}$ and $D_{n}$ such that

$$
\begin{aligned}
P(k) & =C_{n}(-n+k)(\alpha+\beta+n)+D_{n}(-n)(\alpha+\beta+n+k) \\
& =-n C_{n}(\alpha+\beta+n)+k C_{n}(\alpha+\beta+n)-n D_{n}(\alpha+\beta+n)-n k D_{n} \\
& =-k\left[n D_{n}-C_{n}(\alpha+\beta+n)\right]-n(\alpha+\beta+n)\left[C_{n}+D_{n}\right]
\end{aligned}
$$

for every k. Equating the coefficients in front of the degrees of $k$ we form the following system:

$$
\begin{array}{lll}
C_{n}+D_{n} & = & n(\alpha+\beta+n)+\alpha \beta-\beta \delta+\gamma \delta \\
C_{n}(\alpha+\beta+n)-n D_{n} & = & n(\alpha+\beta+n)(\alpha+\beta-\gamma-\delta)+\beta(\alpha-\delta)(\alpha+\beta-\gamma),
\end{array}
$$

whose solution is:

$$
\begin{aligned}
C_{n} & =\frac{(n+\alpha+\beta-\gamma)(n+\alpha-\delta)(n+\beta)}{(\alpha+\beta+2 n)} \\
D_{n} & =\frac{(\alpha+n)(\gamma+n)(n+\beta+\delta)}{(\alpha+\beta+2 n)} .
\end{aligned}
$$

And finally substituting in (15) the expression for $F(x)$ we obtain that

$$
A_{n}(x)=-\frac{n(n+\beta)(n+\alpha+\beta-\delta)(n-\gamma+\alpha+\beta)}{(\alpha+\beta+2 n)(x+\gamma+\delta+1)(x+\beta+\delta+1)(x+\gamma+1)(x+\alpha+1)}
$$

$B_{n}(x)=$

$-\frac{n[(\alpha+\beta+2 n)(\alpha+\beta+n+x+1)(x+\gamma+\delta+1)+(\beta+n)(n+\alpha-\delta)(n+\alpha+\beta-\gamma)]}{(\alpha+\beta+2 n)(x+\gamma+\delta+1)(x+\beta+\delta+1)(x+\gamma+1)(x+\alpha+1)}$

Since the first derivative of any polynomial is can be expressed as a linear combination of differences from the abobe theorem one can conclude that the above polynomial sets satisfy a relation of the form

$$
p_{n}^{\prime}(\lambda(x))=A_{n}(x) p_{n-1}(\lambda(x))-B_{n}(\lambda(x)) p_{n}(\lambda(x)) .
$$


3. Discriminants. The discriminant of a polynomial $p_{n}(x)$ with zeros $x_{n, 1}, x_{n, 2}, \ldots x_{n, n}$ and a leading coefficient $\gamma_{n}$ is given by

$$
D\left(p_{n}(x)\right):=\gamma_{n}^{2 n-2} \prod_{1 \leq j<k \leq n}\left(x_{n, j}-x_{n, k}\right)^{2}=(-1)^{\frac{n(n-1)}{2}} \gamma_{n}^{n-2} \prod_{j=1}^{n} p_{n}^{\prime}\left(x_{n, j}\right) .
$$

In this section we prove that if a sequence of orthogonal polynomials satisfy an equation of the form (2) then the absolute value of its discriminant can be evaluated in terms of the functions $A_{n}(x)$ and its recurrence coefficients. Let us first state a lemma due to Schur [10]

Lemma 3.1. Let $\left\{p_{n}(x)\right\}$ be a sequence of polynomials, generated by the initial conditions

$$
p_{0}(x)=1, \quad p_{1}(x)=a_{1} x+b_{1},
$$

and the three term recurrence relation

$$
p_{n}(x)=\left(a_{n} x+b_{n}\right) p_{n-1}(x)-c_{n} p_{n-2}(x) .
$$

Let $\left\{x_{n, k}\right\}_{k=1}^{n}$ be the zeros of $p_{n}(x)$, then

$$
\prod_{i=1}^{n} p_{n-1}\left(x_{n, i}\right)=(-1)^{\frac{n(n-1)}{2}}\left[\prod_{i=1}^{n} a_{i}^{n-i}\right] \times\left[\prod_{i=2}^{n}\left(\frac{c_{i}}{a_{i}}\right)^{i-1}\right] .
$$

Let us prove a lemma analog to the stated above Schur's lemma.

Lemma 3.2. Let $R_{n}(\lambda(x))$ be a sequence of polynomials generated by

$$
R_{0}(x)=1 \quad R_{1}(x)=a_{1} \lambda(x)+b_{1}
$$

and the three term recurrence relation

$$
R_{n}(\lambda(x))=\left(a_{n} \lambda(x)+b_{n}\right) R_{n-1}(\lambda(x))-c_{n} R_{n-1}(\lambda(x)),
$$

where

$$
\lambda(x)=x(x+\gamma+\delta+1) .
$$

Let $R_{n}\left(\lambda_{n, i}(x)\right)=0 \quad$ for $\quad i=1, \ldots, n \quad$ and

$$
\lambda_{n, i}=x_{n, i, k}\left(x_{n, i, k}+\gamma+\delta+1\right) \quad k=1,2,
$$

then

$$
\prod_{i=1}^{n} R_{n-1}\left(\lambda\left(x_{n, i, 1}\right)\right) R_{n-1}\left(\lambda\left(x_{n, i, 2}\right)\right)=\prod_{i=1}^{n} R_{n-1}\left(\lambda_{n, i}\right)^{2},
$$

or in other words

$$
\prod_{i=1}^{n} R_{n-1}\left(\lambda\left(x_{n, i, 1}\right)\right) R_{n-1}\left(\lambda\left(x_{n, i, 2}\right)\right)=\left[\prod_{i=1}^{n} a_{i}^{2(n-i)}\right] \times\left[\prod_{j=2}^{n}\left(\frac{c_{i}}{a_{i}}\right)^{2(i-1)}\right] .
$$


Proof. Since $R_{n}(\lambda(x))$ is a polynomial of degree $2 n$ of $x$ it can be expressed as:

$$
R_{n}(\lambda(x))=\gamma_{n} \prod_{j=1}^{n}\left(\lambda-\lambda_{n, j}\right)=\gamma_{n} \prod_{j=1}^{n}\left(x-x_{n, j, 1}\right)\left(x+x_{n, j, 1}+\gamma+\delta+1\right)
$$

Therefore

$$
\begin{gathered}
\prod_{i=1}^{n} R_{n-1}\left(x_{n, i, 1}\right) R_{n-1}\left(x_{n, i, 2}\right) \\
=\gamma_{n-1}^{2 n}\left[\prod_{i=1}^{n} \prod_{j=1}^{n-1}\left(x_{n, i, 1}-x_{n-1, j, 1}\right)\left(x_{n, i, 1}+x_{n-1, j, 1}+\gamma+\delta+1\right)\right] \\
\times\left[\prod_{i=1}^{n} \prod_{j=1}^{n-1}\left(x_{n, i, 2}-x_{n-1, j, 1}\right)\left(x_{n, i, 2}+x_{n-1, j, 1}+\gamma+\delta+1\right)\right] \\
=\gamma_{n-1}^{2 n} \prod_{i=1}^{n} \prod_{j=1}^{n-1}\left(x_{n, i, 1}-x_{n-1, j, 1}\right)^{2}\left(x_{n, i, 1}+x_{n-1, j, 1}+\gamma+\delta+1\right)^{2} \\
=\prod_{i=1}^{n} R_{n-1}\left(\lambda_{i, n}\right)^{2}=\left[\prod_{i=1}^{n} a_{i}^{2(n-i)}\right] \times\left[\prod_{j=2}^{n}\left(\frac{c_{i}}{a_{i}}\right)^{2(i-1)}\right] .
\end{gathered}
$$

Here we used

$$
x_{n, i, 2}=-x_{n, i, 1}-\gamma-\delta-1
$$

and Schur's lemma.

THEOREM 3.1. Let $R_{n}(\lambda(x))$ be a sequence of polynomials satisfying

$$
R_{n}^{\prime}(\lambda(x))=A_{n}(x) R_{n-1}(\lambda(x))-B_{n}(x) R_{n}(\lambda(x)),
$$

then

$$
D^{2}\left(R_{n}(\lambda(x))=\left[\prod_{i=1}^{n} A_{n}\left(x_{n, i, 1}\right) A_{n}\left(-x_{n, i, 1}-\gamma-\delta-1\right) a_{i}^{4 n-2 i-4}\right] \times\left[\prod_{i=2}^{n}\left(\frac{c_{i}}{a_{i}}\right)^{2 i-2}\right] .\right.
$$

Proof. From the definition, and the fact that $R_{n}\left(\lambda\left(x_{n, j}\right)\right)=0$, it follows that

$$
\begin{aligned}
D^{2}\left(R_{n}(\lambda(x))\right. & =\gamma_{n}^{4 n-4} \prod_{i=1}^{n} A_{n}\left(x_{n, i, 1}\right) A_{n}\left(-x_{n, i, 1}-\gamma-\delta-1\right) R_{n-1}^{2}\left(\lambda_{n, i}\right) \\
& =\left[\prod_{i=1}^{n} A_{n}\left(x_{n, i, 1}\right) A_{n}\left(-x_{n, i, 1}-\gamma-\delta-1\right) a_{i}^{4 n-2 i-4}\right] \times\left[\prod_{i=2}^{n}\left(\frac{c_{i}}{a_{i}}\right)^{2 i-2}\right] .
\end{aligned}
$$

$\square$ 
4. Some resultants. Let $p_{n}(\lambda(x))=q_{2 n}(x)$ is of precise degree $n$ w.r.t. the variable $\lambda(x)$ that is $q_{2 n}(x)$ is of exact degree $2 n$ w.t.r. $x$. Let $T q_{2 n}(x)$ is of precise degree $2 n-2$ w.r.t. the variable $x$ and both $q_{n}(x)$ and $T q_{n}(x)$ have the same sign. Consider the following resultant:

$$
D^{2}\left(p_{n}(\lambda(x)), T\right)=\gamma_{n}^{-2} R\left\{q_{2 n}(x), T\left(q_{2 n}(x)\right)\right\}=\gamma_{n}^{2 n-4} \prod_{i=1}^{n} T\left(q_{2 n}\left(x_{n, i, 1}\right) T\left(q_{2 n}\left(x_{n, i, 2}\right)\right),\right.
$$

where $x_{n, i, 1}$ and $x_{n, i, 2}$ are the zeros of $q_{2 n}(x)$ which satisfy :

$$
x_{n, i, 2}=-x_{n, i, 1}-\gamma-\delta-1
$$

by Vietta's formula.

Let us state some theorems that show the way we can compute resultants of the above type.

THEOREM 4.1. Let $R_{n}(\lambda(x))$ be a sequence of polynomials satisfying

$$
\Delta R_{n}(\lambda(x))=\frac{\delta R_{n}(\lambda(x))}{\delta(\lambda(x))}=A_{n}(x) R_{n-1}(\lambda(x))-B_{n}(x) R_{n}(\lambda(x))
$$

where

$$
\delta R_{n}(\lambda(x))=R_{n}(\lambda(x+1))-R_{n}(\lambda(x)),
$$

and

$$
\delta \lambda(x)=\lambda(x+1)-\lambda(x) .
$$

then

$$
\begin{aligned}
D^{2}\left(R_{n}, \lambda(x), \Delta, 1\right)= & {\left[\prod_{i=1}^{n} A_{n}\left(x_{n, i, 1}\right) A_{n}\left(-x_{n, i, 1}-\gamma-\delta-1\right) a_{i}^{4 n-2 i-4}\right] } \\
& \times\left[\prod_{i=2}^{n}\left(\frac{c_{i}}{a_{i}}\right)^{2 i-2}\right] .
\end{aligned}
$$

Proof. The proof of this theorem resembles the proof in the Ismail's papers, however we apply here the analogue of the Shur's lemma.

REMARK. These quantities of the above resultants are the same if we use the divided difference operator with the backwards differences.

5. Some resultants. Let us mention that from the above results and the fact that every sequence of polynomials from the Askey scheme satisfy three term recurrence relation the following resultants can be computed.

\subsection{Meixner-Pollaczek polynomials.}

$$
D\left(P_{n}^{(\lambda)}(x), \Delta, i\right)=(2 \sin \theta)^{n(n-1)} \prod_{j=1}^{n}(j+2 \lambda-1)^{j-1} j^{j+2-2 n} .
$$




\subsection{Continuous dual Hahn polynomials.}

$$
D\left(S_{n}\left(x^{2}\right), \Delta, i\right)=\prod_{j=1}^{n} j^{2 j}(a+b+j-1)^{2(j-1)}(a+c+j-1)^{2(j-1)}(b+c+j-1)^{2(j-1)} .
$$

5.3. Dual Hahn polynomials. For the Dual Hahn polynomials formula (34) applies.

$$
D\left(R_{n}(\lambda(x)), \Delta, 1\right)=\prod_{i=1}^{n} \frac{i^{2 i}(N+1+\delta-i)^{2(i-1)}}{((N+1-i)(\gamma+i))^{2(2 n-i-1)}} .
$$

\subsection{Continuous Hahn polynomials.}

$$
\begin{aligned}
D\left(p_{n}(x), \Delta, i\right)= & {\left[\prod_{i=1}^{n} i^{i}(\beta+i)^{i-1}(\alpha+\beta-\gamma+i)^{i-1}(\alpha-\delta+i)^{i-1}(\alpha+i)^{i+1-2 n}\right] } \\
& \times\left[\prod_{i=1}^{n}(\beta+\delta+i)^{i+1-2 n}(\gamma+i)^{i+1-2 n}(\alpha+\beta+n+i)^{n-i}\right]
\end{aligned}
$$

\subsection{Wilson polynomials.}

$$
\begin{aligned}
& D^{2}\left(W_{n}\left(x^{2}\right), \Delta, i\right) \\
= & {\left[\prod_{j=1}^{n} j^{2 j}(b+c+j-1)^{2(j-1)}(b+d+j-1)^{2(j-1)}\right] } \\
& \times\left[\prod_{j=1}^{n}(c+d+j-1)^{2(j-1)}(a+b+j-1)^{2(j-1)}\right] \\
& \times\left[\prod_{j=1}^{n}(a+c+j-1)^{2(j-1)}(a+d+j-1)^{2(j-1)}(a+b+c+d+n+j-2)^{2(n-j)}\right]
\end{aligned}
$$

5.6. Racah polynomials. Here formula (34) applies.

$$
\begin{aligned}
& D^{2}\left(R_{n}(\lambda(x)), \Delta, 1\right) \\
= & {\left[\prod_{i=1}^{n} i^{2 i}(\beta+i)^{2(i-1)}(\alpha+\beta-\gamma+i)^{2(i-1)}(\alpha-\delta+i)^{2(i-1)}\right] } \\
& \times\left[\prod_{i=1}^{n}(\alpha+i)^{2(i+1-2 n)}(\beta+\delta+i)^{2(i+1-2 n)}(\gamma+i)^{2(i+1-2 n)}(\alpha+\beta+n+i)^{2(n-i)}\right] .
\end{aligned}
$$

Acknowledgement. I would like to thank to P. Marinov and A. Andreev for the encouragement.

\section{REFERENCES}

[1] S. S. Bonan And D. S. Clark, Estimates of the Hermite and the Freud polynomials, J. Approx. Theory, 63 (1990), pp. 210-224. 
[2] Y. Chen And M. E. H. Ismail, Ladder operators and differential equations for orthogonal polynomials, J. Phys. A, 30 (1997), pp. 7818-7829.

[3] I. M. Gelfand, M.M. Kapranov and A. V. Zelevinsky, Discriminants, Resultants, and Multidimensional determinants, Birhuser Boston, Boston 1994.

[4] D. HilberT, Über die Discriminante der in Endlichen abbrechenden hypergeometrischen Reihe, J. für die reine und angewandte Mathematik, 103 (1888), pp. 337-345.

[5] M. E. H. Ismail, Discriminants and functions of the second kind of orthogonal polynomials, Result. Math., 34 (1998), pp. 132-149.

[6] M. E. H. IsmaIL, An electrostatic model for zeros of general orthogonal polynomials, Pacific J. Math., 193 (2000), pp. 355-369.

[7] M. E. H. Ismail, Difference equations and quantized discriminants for q-orthogonal polynomials, Advances in Applied Math., 30 (2003), pp. 562-589.

[8] M. E. H. Ismail, I. Nikolova, P. Simeonov, Difference Equations and Discriminants for Discrete orthogonal polynomials - to appear in Ramanujan journal.

[9] R. Koekoek and R. Swarttouw, The Askey-scheme of hypergeometric orthogonal polynomials and its q-analogue, Reports of the Faculty of Technical Mathematics and Informatics no. 98-17, Delft University of Technology, Delft, 1998.

[10] G. Szegő, Orthogonal Polynomials, Fourth Edition, Amer. Math. Soc., Providence, 1975. 
I. NIKOLOVA 